\title{
Effet de la granulation sur les performances de croissance, I'efficacité alimentaire et la viabilité des lapereaux en condition d'élevage tropical
}

\author{
M. Kpodékon ${ }^{1,2}$ A.K.I. Youssao ${ }^{1 *}$ G.B. Koutinhouin ${ }^{1}$ \\ I.L. Baba ${ }^{1}$ J.M. Dessou ${ }^{1}$ Y. Djago ${ }^{2}$
}

\begin{abstract}
Mots-clés
Lapin - Aliment composé pour animaux - Croissance - Viabilité Bénin.
\end{abstract}

\begin{abstract}
Résumé
Cent quarante-quatre lapereaux de race locale ont été utilisés à partir du sevrage (entre 34 et 36 jours d'âge) pour analyser leur croissance, leur efficacité alimentaire et leur viabilité, en fonction de la présentation de l'aliment : farine ou granulé. L'expérimentation s'est déroulée sur trois fermes (Cecuri, Gava et Ahinadjè), à raison de 48 lapereaux par ferme, répartis en deux lots de 24 lapereaux : un lot expérimental nourri avec un aliment granulé et un lot témoin nourri avec le même aliment mais sous forme de farine. Le poids et la consommation ont été mesurés hebdomadairement durant les huit semaines d'engraissement et la mortalité a été contrôlée quotidiennement. Pendant la durée d'engraissement (56 jours), les lapereaux nourris avec l'aliment granulé ont eu une vitesse de croissance $(24 \mathrm{~g} / \mathrm{j})$ plus élevée $(\mathrm{P}<0,001)$ que celle des lapereaux nourris avec I'aliment farineux $(22 \mathrm{~g} / \mathrm{j})$. Pendant les quatre premières semaines, l'aliment granulé a été davantage consommé (+ 18 p. 100), moins rejeté (- 77 p. 100), avec un indice de consommation plus faible (- 20 p. 100), que l'aliment farineux $(\mathrm{P}<0,001)$. L'indice de consommation $(5,2)$ et les quantités d'aliment consommées $(92$ g) ont été identiques pendant les quatre dernières semaines $(P>0,05)$. En revanche, I'aliment granulé a été moins refusé $(P<0,001)$ que I'aliment farineux (1 g vs $4 \mathrm{~g})$. Enfin, dans les lots des lapereaux nourris au granulé, le taux de mortalité a été de 15 p. 100, contre 46 p. 100 chez les lapereaux nourris à l'aliment farineux.
\end{abstract}

\section{INTRODUCTION}

L'alimentation est l'un des facteurs les plus importants à maitriser pour le développement de l'élevage du lapin (Oryctolagus cuniculus) sous les tropiques en général et au Bénin en particulier. Ainsi, depuis une dizaine d'années, le Centre cunicole de recherche et d'information (Cecuri) mène des travaux pour améliorer l'alimentation afin d'assurer une bonne productivité et une meilleure rentabilité économique des élevages cunicoles qui sont aujourd'hui

\footnotetext{
1. Ecole polytechnique d'Abomey-Calavi, Laboratoire de recherche en biologie appliquée, UR Cunicole et cavicole, Cotonou, Bénin.

2. Centre cunicole de recherche et d'information, Cotonou, Bénin.

* Auteur pour la correspondance

Ecole polytechnique d'Abomey-Calavi, département de production et santé animales, 01 BP 2009, Cotonou, Bénin.

Tél. : +22995285988; fax : +22921360199

E-mail : iyoussao@yahoo.fr
}

en pleine expansion au Bénin. Au Cecuri, il faut 56 jours d'engraissement, à partir du sevrage qui se fait à l'âge de 35 jours, pour atteindre un poids moyen de $1920 \mathrm{~g}$ chez les lapins nourris à l'aliment granulé, et de $1822 \mathrm{~g}$ chez les lapins nourris avec un aliment farineux (8). Dans les pays tempérés, la vitesse de croissance peut atteindre 48 à $51 \mathrm{~g} /$ jour $(\mathrm{j})$, correspondant à un poids à l'abattage d'environ $2700 \mathrm{~g}$, pour des lapins sevrés à 35 jours et nourris avec un aliment granulé (12). Selon Kpodékon et coll. (7), les lapins d'engraissement du Cecuri, soumis pendant huit semaines à un aliment composé farineux local et à un aliment granulé importé, ont abouti à des gains moyens quotidiens (GMQ) de 23,4 g/j pour l'aliment farineux et de $28,5 \mathrm{~g} / \mathrm{j}$ pour l'aliment granulé. En climat tropical (Guadeloupe), la croissance est similaire aux valeurs du Cecuri (3). Dans ces expériences, les aliments farineux et granulés n'avaient pas la même composition centésimale, ce qui n'a pas permis d'évaluer réellement l'effet de la granulation. Le but de cette étude a été de comparer l'ingestion, la croissance et la santé de lapins sevrés et nourris soit avec un aliment granulé, soit avec le même aliment non granulé (en farine). 


\section{MATERIEL ET METHODES}

\section{Cadre d'étude}

Les expérimentations se sont déroulées pendant huit semaines (du 24 février au 12 mai 2005) dans trois élevages :

- le Cecuri, unité d'application de l'Ecole polytechnique d'Abomey-Calavi, situé sur le campus universitaire d'Abomey-Calavi, à $600 \mathrm{~m}$ environ de la route inter-Etats (Bénin-Niger);

- la ferme cunicole Gava-lapins, située à Cococodji, à environ $16 \mathrm{~km}$ de l'université d'Abomey-Calavi, sur la route inter-Etats (Bénin-Togo);

- la ferme cunicole Ahinadjè-lapins, située dans le village de Hêvié, à environ 20 km de la route inter-Etats (Bénin-Niger).

Ces trois élevages étaient localisés dans la commune d'AbomeyCalavi, située dans le département de l'Atlantique. Ce département bénéficie de conditions climatiques de type subéquatorial, caractérisé par deux saisons de pluies : la grande (avril à juillet) et la petite (septembre à novembre). Ces deux saisons sont intercalées par des saisons sèches. La pluviométrie moyenne est voisine de $1200 \mathrm{~mm}$ par an. Les températures moyennes mensuelles varient entre 27 et $31{ }^{\circ} \mathrm{C}$ et l'humidité relative de l'air fluctue entre 65 p. 100, de janvier à mars, et 97 p. 100, de juin à juillet.

\section{Schéma expérimental}

Cent quarante-quatre lapereaux de race locale ont été utilisés à partir du sevrage (entre 34 à 36 jours d'âge), à raison de 48 lapereaux par ferme. Dans chaque ferme, les lapereaux ont été répartis en fonction de leur poids au sevrage en deux lots (expérimental et témoin) de 24 lapereaux, et groupés dans huit cages de trois animaux par lot. Les cages métalliques $(75 \mathrm{~cm} \times$ $45 \mathrm{~cm} \times 30 \mathrm{~cm}$ ) ont été disposées en flat-deck dans des bâtiments ventilés naturellement et éclairés par la lumière du jour. Chaque cage était munie :

- d'un système de récupération pour recueillir les aliments gaspillés et éviter qu'ils ne soient mélangés aux crottes et à l'urine ;

- d'une mangeoire en tôle galvanisée accrochée à l'intérieur de la cage ;

- d'un abreuvoir en système de vases communicants et constitué d'un bac à eau muni de flotteur interne de stabilisation du niveau d'eau, d'un long tuyau et de tétines.

Les lots expérimentaux ont reçu l'aliment granulé (aliment essai) et les lots témoins l'aliment farineux, de même composition centésimale. Les aliments farineux et granulés ont été distribués ad libitum chaque jour à $9 \mathrm{~h}$ et à $16 \mathrm{~h}$. Les animaux des deux lots (expérimental et témoin) ont reçu un complément de fourrage vert constitué de feuilles d'Elaeis guineensis (récoltées chaque jour) pour combler l'insuffisance de la cellulose. Ces feuilles sont composées de 92,9 p. 100 de matière sèche, 9,9 p. 100 de matière azotée totale (MAT), 4,2 p. 100 de matière grasse et 19,0 p. 100 de cellulose (4). Le tableau I montre la composition des aliments granulés et farineux. La teneur en cellulose brute des aliments consommés et des refus a été déterminée au laboratoire de la Direction de l'alimentation et de la nutrition appliquée. Le dosage de l'azote total a été fait par la méthode de Kjeldahl et la valeur de la MAT a ensuite été déduite en multipliant la teneur en azote par le coefficient 6,25. La cellulose brute a été déterminée par la méthode de Weende. L'énergie digestible de l'aliment a été calculée à partir de la formulation, en additionnant l'énergie fournie par chaque matière première entrant dans sa composition. Avant la constitution des lots, Trisulmycine Forte (sulfadiazine 66,7 g et triméthoprime 13,3 g) a été utilisée dans l'eau de boisson pour prévenir la coccidiose à la dose de $2 \mathrm{mg} / \mathrm{kg}$ de poids vif pendant trois jours.

\section{Collecte des données}

Après une pesée individuelle des animaux à J0, jour du démarrage de l'expérience, les animaux ont été pesés hebdomadairement à jour fixe durant les huit semaines d'engraissement. Les quantités d'aliments distribuées, consommées et refusées ont été quotidiennement enregistrées. Au cours de l'essai, les animaux morts ont été enregistrés et retirés des cages, et un diagnostic post mortem a été fait pour chacun d'eux.

\section{Traitement des données}

Pour le traitement des données, les poids hebdomadaires ont été enregistrés et les différents gains moyens quotidiens ont été calculés. L'indice de consommation a été déterminé en fonction du nombre de lapin-jours (nombre de jours pour lesquels chaque lapin a réellement consommé l'aliment dans la cage commune). Les analyses statistiques ont ensuite été réalisées avec le logiciel SAS (11). Un modèle linéaire comprenant les effets fixes de l'aliment (granulé et standard) et de la ferme (Cecuri, Gava et Ahinadjè) a été ajusté aux données. Les effets de l'interaction entre l'aliment et la ferme n'ont pas été significatifs sur le poids et le gain moyen quotidien $(\mathrm{P}>0,05)$ et, par conséquent, n'ont pas été pris en compte dans le modèle. L'analyse de la variance a été obtenue par la procédure des modèles linéaires généralisés (Proc GLM). Les moyennes des moindres carrés ont ensuite été estimées et comparées par le test de $t$. La comparaison entre les différents taux de mortalité a été faite par le test du chi-2 en utilisant la procédure Catmod de SAS (11) et en intégrant dans le modèle l'aliment, la ferme et l'interaction entre l'aliment et la ferme.

\section{Tableau I}

Composition centésimale et chimique des aliments granulés et farineux

\begin{tabular}{lcc} 
& Aliment granulé & Aliment farineux \\
\hline Matières premières (\%) & & \\
Maïs & 5,0 & 5,0 \\
Tourteau de palmiste & 29,0 & 29,0 \\
Tourteau de coton & 16,0 & 16,0 \\
Tourteau de soja & 7,0 & 7,0 \\
Son de blé & 20,5 & 20,5 \\
Son de riz & 15,0 & 15,0 \\
Coquille d'huître & 2,5 & 2,5 \\
Sel & 0,5 & 0,5 \\
Sciure de bois & 4,0 & 4,0 \\
Total & 99,5 & 99,5 \\
& & \\
Composition chimique & & 89,2 \\
Matière sèche (\%) & 88,4 & 10,99 \\
Energie digestible ${ }^{1}$ & 10,94 & 19,1 \\
(MJ/kg de MS) & & 18,9 \\
Cellulose (\% de MS) & 19,8 & \\
Matière azotée totale & 18,8 & \\
(\% de MS) & & \\
\hline
\end{tabular}

${ }^{1}$ L'énergie digestible de l'aliment a été obtenue à partir de la somme de l'énergie fournie par chaque matière première entrant dans sa composition. 


\section{RESULTATS}

\section{Effet des aliments granulés et farineux sur les performances de croissance}

Au début de l'expérimentation, aucune différence significative n'a été observée entre le poids au sevrage des deux lots nourris (527 g). La différence pondérale est apparue à partir du septième jour, jusqu'à la fin de l'expérimentation $(\mathrm{P}<0,01)$ en faveur de l'aliment granulé : les lapereaux nourris aux granulés pesaient $1793 \mathrm{~g}$ à la fin de l'expérimentation, alors que ceux nourris à l'aliment farineux pesaient $1480 \mathrm{~g}$, soit un écart de 33 p. 100 équivalent à un gain de 13 à 15 jours d'engraissement.

Pendant les quatre premières semaines, les lapereaux nourris aux granulés ont eu un GMQ significativement plus élevé (30 g/j) que ceux nourris à l'aliment farineux $(24 \mathrm{~g} / \mathrm{j})(\mathrm{P}<0,001)$. De même, pendant les quatre dernières semaines, cette différence de gain a été maintenue $(\mathrm{P}<0,05)$ avec un GMQ de 17 et de $14 \mathrm{~g} / \mathrm{j}$, respectivement pour les lapereaux nourris aux granulés et ceux nourris à l'aliment farineux. Pour toute la durée d'engraissement, les lapereaux nourris aux granulés ont eu un GMQ (24 g/j) significativement plus élevé $(\mathrm{P}<0,001)$ que celui des lots ayant reçu l'aliment farineux $(22 \mathrm{~g} / \mathrm{j})$.

Le poids des lapereaux de la ferme Gava et du Cecuri a été plus élevé $(\mathrm{P}<0,001)$ que celui des lapereaux d'Ahinadjè (tableau II). Par rapport au poids initial, le gain total de poids pendant la période d'engraissement a été de 1279,1325 et $1200 \mathrm{~g}$ pour les granulés, et 893, 951 et $1022 \mathrm{~g}$ pour l'aliment farineux, respectivement chez les lapereaux du Cecuri, de Gava et d'Ahinadjè.

\section{Consommation d'aliment et indice de consommation}

Pendant les quatre premières semaines, l'aliment granulé a été plus consommé (+ 18 p. 100), moins rejeté (- 77 p. 100) avec un indice de consommation plus faible (- 20 p. 100) que l'aliment farineux ( $\mathrm{P}<0,001$, tableau III). L'indice de consommation $(5,2)$ et les quantités d'aliment consommées (92 g) ont été identiques pendant les quatre dernières semaines $(\mathrm{P}>0,05)$. En revanche, l'aliment granulé a été moins rejeté $(\mathrm{P}<0,001 \mathrm{~g})$ que l'aliment farineux $(1 \mathrm{~g}$ vs $4 \mathrm{~g})$.

Les quantités d'aliment consommées n'ont pas varié d'une ferme à l'autre. Il en a été de même pour l'indice de consommation des quatre dernières semaines (tableau III). Toutefois, pendant les quatre premières semaines, les lapereaux d'Ahinadjè ont eu un indice de consommation plus élevé que ceux du Cecuri et de Gava (22 p. 100), et la quantité d'aliment rejetée la plus faible (1,7 g) a été obtenue à Gava. La teneur en cellulose brute des refus de l'aliment farineux a été de 38,4 p. 100 contre 19,21 p. 100 pour l'aliment granulé. Ces refus ont été composés à 75 p. 100 de sciure de bois.

\section{Morbidité et mortalité}

Le taux de mortalité des lots nourris avec l'aliment farineux $(45,8$ p. 100) a été plus élevé $(\mathrm{P}<0,001)$ que celui des lots nourris avec l'aliment granulé (15,3 p. 100, tableaux IV et V). En revanche, le taux de mortalité de l'ensemble des animaux pour les deux lots confondus n'a pas différé significativement d'une ferme à l'autre (P > 0,05). Le taux de mortalité au Cecuri a été de 31,3 p. 100 et ceux d'Ahinadjè et de Gava ont été respectivement de 31,3 et 29,2 p. 100.

Au Cecuri, aucune différence significative $(P=0,057)$ n'a été observée entre le taux de mortalité des lapereaux nourris à l'aliment granulé $(16,7$ p. 100) et celui des lapereaux nourris à l'aliment farineux (41,7 p. 100). En revanche, le taux de mortalité a été plus élevé $(\mathrm{P}<0,05)$ chez les lapereaux nourris à l'aliment farineux que chez ceux nourris à l'aliment granulé à Gava $(50,0$ vs 12,5 p. 100) et à Ahinadjè (45,8 vs 16,7 p. 100).

\section{Tableau II}

Poids vif ${ }^{1}$ et croissance des lapereaux en fonction de la forme de présentation de l'aliment : en farine ou granulé

\begin{tabular}{|c|c|c|c|c|c|c|c|c|}
\hline \multirow[t]{2}{*}{ Variable } & \multicolumn{2}{|c|}{ Aliment } & \multicolumn{3}{|c|}{ Ferme } & \multicolumn{2}{|c|}{ Test de signification ${ }^{2}$} & \multirow[t]{2}{*}{ ETR } \\
\hline & Farineux & Granulé & Ahinadjè & Cecuri & Gava & Aliment & Ferme & \\
\hline P0 (g) & $528^{a}$ & $526^{a}$ & $459^{a}$ & $569 \mathrm{~b}$ & $553^{b}$ & NS & $* * *$ & 84 \\
\hline P7 (g) & $644^{\mathrm{a}}$ & $761^{b}$ & $626^{a}$ & $737^{b}$ & $745^{b}$ & $* * *$ & $* * *$ & 114 \\
\hline P14 (g) & $767^{a}$ & $951^{b}$ & $769^{a}$ & $909 \mathrm{~b}$ & $898^{b}$ & $* * *$ & $* * *$ & 141 \\
\hline P21 (g) & $946^{\mathrm{a}}$ & $1159 \mathrm{~b}$ & $918^{a}$ & $1112^{b}$ & $1128^{b}$ & $* * *$ & $* * *$ & 178 \\
\hline P28 (g) & $1105^{a}$ & $1343^{b}$ & $1103^{a}$ & $1239 b$ & $1328^{c}$ & $* * *$ & $* * *$ & 191 \\
\hline P35 (g) & $1228^{a}$ & 1492 b & $1216^{a}$ & $1385^{b}$ & $1479 \mathrm{~b}$ & $* * *$ & $* * *$ & 212 \\
\hline P42 (g) & $1378^{a}$ & $1619 b$ & $1380^{a}$ & $1495^{b}$ & $1620^{c}$ & $* * *$ & $* * *$ & 224 \\
\hline P49 (g) & $1452^{a}$ & $1716^{b}$ & $1471^{a}$ & 1597 b & $1685^{b}$ & $* *$ & $* * *$ & 260 \\
\hline P56 (g) & $1480^{a}$ & $1793^{b}$ & $1548^{a}$ & $1662^{b}$ & $1699 \mathrm{~b}$ & $* * *$ & $*$ & 317 \\
\hline GMQ40 (g/j) & $24,4^{\mathrm{a}}$ & $30,1 \mathrm{~b}$ & $25,9^{a}$ & 26,8 ab & $29,0^{b}$ & $* * *$ & $*$ & 5,7 \\
\hline GMQ85 (g/j) & $14,0^{a}$ & $16,7^{b}$ & $14,7^{\mathrm{a}}$ & $15,7^{a}$ & $15,6^{\mathrm{a}}$ & * & NS & 5,6 \\
\hline GMQ80 (g/j) & $20,9^{a}$ & $24,4^{b}$ & $21,3^{a}$ & $21,7^{a}$ & $24,9 \mathrm{~b}$ & $* * *$ & $*$ & 5,3 \\
\hline
\end{tabular}

${ }^{1}$ Les valeurs sont les moyennes ajustées (moindres carrés)

${ }^{2}$ L'interaction ferme $\mathrm{x}$ aliment est non significative

a,b,c Les moyennes suivies de lettres différentes diffèrent significativement au seuil de $5 \%$

$* \mathrm{P}<0,05 ; * * \mathrm{P}<0,01 ; * * * \mathrm{P}<0,001 ; \mathrm{NS}:$ non significatif

$\mathrm{Pi}$ : poids au i jour d'engraissement; GMG ij : gain moyen quotidien entre la $\mathrm{j}$ et la $\mathrm{i}^{\mathrm{e}}$ semaine d'engraissement

ETR : écart-type résiduel 


\section{Tableau III}

Quantités d'aliment consommées et rejetées, et indice de consommation des lapereaux en fonction de la forme de présentation des aliments dans les différentes fermes

\begin{tabular}{|c|c|c|c|c|c|c|c|c|}
\hline \multirow[t]{2}{*}{ Variables } & \multicolumn{2}{|c|}{ Aliment } & \multicolumn{3}{|c|}{ Ferme } & \multicolumn{2}{|c|}{ Test de signification 2} & \multirow[t]{2}{*}{ ETR } \\
\hline & Farineux & Granulé & Ahinadjè & Cecuri & Gava & Aliment & Ferme & \\
\hline Aliment consommé 1 (g) & $77,5^{\text {a }}$ & $65,6^{b}$ & $82,1^{a}$ & $66,9^{a}$ & $56,6^{\mathrm{a}}$ & $* * *$ & NS & 16,7 \\
\hline Aliment refusé 1 (g) & $0,9^{a}$ & $3,8 \mathrm{~b}$ & $2,4^{\mathrm{a}}$ & $2,9^{a}$ & $1,7 \mathrm{~b}$ & $* * *$ & $* * *$ & 1,6 \\
\hline Indice de consommation 1 & $2,9^{\mathrm{a}}$ & $3,6^{b}$ & $3,7^{\text {a }}$ & $3,2^{b}$ & $2,9 b$ & $* * *$ & $* *$ & 1,4 \\
\hline Aliment consommé 2 (g) & $87,6^{a}$ & $96,9^{a}$ & $97,7^{a}$ & 89,2 a & $89,8^{a}$ & NS & NS & 34,8 \\
\hline Aliment refusé 2 (g) & $1,0^{\mathrm{a}}$ & $4,3^{b}$ & $2,7^{\mathrm{a}}$ & $3,0^{\mathrm{a}}$ & $2,2^{b}$ & $* * *$ & $* * *$ & 1,2 \\
\hline Indice de consommation 2 & 5,0 & 5,4 & 5,4 & 5,3 & 4,8 & NS & NS & 2,0 \\
\hline
\end{tabular}

Aliment consommé 1 : aliment quotidiennement consommé pour les quatre premières semaines (35 à 63 jours)

Aliment consommé 2 : aliment quotidiennement consommé pour les quatre dernières semaines (64 à 81 jours)

ETR : écart-type résiduel

${ }^{\mathrm{a}, \mathrm{b}}$ Les moyennes suivies de lettres différentes diffèrent significativement au seuil de $5 \%$

$* \mathrm{P}<0,05 ; * * \mathrm{P}<0,01 ; * * * \mathrm{P}<0,001 ; \mathrm{NS}$ : non significatif

\section{Tableau IV}

Signification des sources de variation de la mortalité des lapereaux au cours de l'engraissement

\begin{tabular}{lcrc} 
Source de variation & DDL & Chi-2 & Signification \\
\hline Intercept & 1 & 21,61 & $* * *$ \\
Ferme & 2 & 0,04 & NS \\
Aliment & 1 & 14,61 & $* * *$ \\
Interaction Ferme x Aliment & 2 & 0,47 & NS
\end{tabular}

DDL : degré de liberté

$* * * \mathrm{P}<0,001 ; \mathrm{NS}$ : non significatif

\section{Tableau V}

Taux de mortalité des lapereaux au cours de l'engraissement

\begin{tabular}{|c|c|c|c|}
\hline Source de variation & Effectif & $\begin{array}{l}\text { Nb. de } \\
\text { morts }\end{array}$ & $\begin{array}{c}\text { Taux de } \\
\text { mortalité (\%) }\end{array}$ \\
\hline \multicolumn{4}{|l|}{ Ferme } \\
\hline Cecuri & 48 & 15 & $31,3^{a}$ \\
\hline Gava & 48 & 14 & $29,2^{\text {a }}$ \\
\hline Ahinadjè & 48 & 15 & $31,3^{a}$ \\
\hline \multicolumn{4}{|l|}{ Aliment } \\
\hline Granulé & 72 & 11 & $15,3^{a}$ \\
\hline Farineux & 72 & 33 & $45,8^{b}$ \\
\hline \multicolumn{4}{|l|}{ Interaction } \\
\hline Cecuri x Granulé & 24 & 4 & $16,7^{a b}$ \\
\hline Cecuri x Farineux & 24 & 10 & $41,7^{a b}$ \\
\hline Gava x Granulé & 24 & 3 & $12,5^{b}$ \\
\hline Gava x Farineux & 24 & 12 & $50^{a}$ \\
\hline Ahinadjè x Granulé & 24 & 4 & 16,2 ab \\
\hline Ahinadjè $x$ Farineux & 24 & 11 & $45,8^{a b}$ \\
\hline
\end{tabular}

Les taux de mortalité intra-classes suivis d'au moins une lettre commune ne diffèrent pas significativement au seuil de $5 \%$
Pour les causes de mortalité, 36 cas d'entérite (inflammation du caecum et de l'iléon) ont été enregistrés dont 30 dans le lot « farine » et six dans le lot « granulé ». Les maladies respiratoires (inflammation des poumons, densification des lobes, inflammation du sinus) ont été uniquement rencontrées dans le lot témoin avec six cas. Les causes de deux cas de mortalité n'ont pas pu être identifiées (absence de diarrhées et de signes cliniques apparents).

\section{Aspects économiques}

Les coûts et bénéfices bruts ont été calculés en tenant compte uniquement du facteur aliment, les coûts fixes et les autres coûts variables étant les mêmes (tableau VI). Le prix du kilogramme de poids vif de lapin a été égal à $1000 \mathrm{Fcfa}$ et le coût de la granulation a été de 40 Fcfa. Par rapport à l'aliment farineux, une marge bénéficiaire de 245 Fcfa par lapin a été obtenue à l'issue de cette étude, confirmant la rentabilité économique de l'aliment granulé.

\section{DISCUSSION}

\section{Performances pondérales et consommation d'aliment}

L'aliment granulé a été plus consommé que l'aliment farineux, ce qui a entraîné une croissance supérieure dans le lot " granulé ». Ces deux aliments étant de même composition, les plus faibles performances du lot «farine »seraient liées à la forme de présentation de l'aliment. En effet, la présentation en farine favorise le tri des différentes particules alimentaires par les animaux. Les travaux de Goby et Rochon (6) sur la digestibilité et l'impact du tri alimentaire montrent que les lapins délaissent les copeaux de bois pourtant riches en fibres. Selon Kpodékon et coll. (7), la faible croissance pondérale des lapereaux nourris à l'aliment farineux est essentiellement due à une sous-consommation alimentaire.

Ainsi, la granulation de l'aliment a permis d'obtenir une croissance supérieure à celle induite par l'aliment farineux (24,4 vs 20,9 g/j) entre le sevrage et l'abattage. Cette tendance a été similaire à celle enregistrée par Kpodékon et coll. (8), avec un aliment granulé de même formule que celle utilisée dans la présente étude, comparé avec un aliment farineux de composition différente de celle de l'aliment granulé (28,3 vs 24,6 g/j). 
Tableau VI

Coûts et bénéfices bruts à la fin de l'engraissement

\begin{tabular}{lcr}
$\begin{array}{l}\text { Paramètres } \\
\text { économiques }\end{array}$ & Traitement & $\begin{array}{c}\text { Ensemble } \\
\text { des 3 élevages }\end{array}$ \\
$\begin{array}{l}\text { Prix du kilogramme } \\
\text { de l'aliment (Fcfa) }\end{array}$ & Farine & 110 \\
$\begin{array}{l}\text { Quantité globale } \\
\text { d'aliment utilisée } \\
\text { par les animaux (kg) }\end{array}$ & Farine & 150 \\
$\begin{array}{l}\text { Prix de la quantité } \\
\text { d'aliment utilisée }\end{array}$ & Granulé & 71 \\
par les animaux (Fcfa) & Farine & 700 \\
Gain de poids vif (kg) & Granulé & 14932 \\
& & \\
$\begin{array}{l}\text { Prix de vente } \\
\text { du gain de poids vif (Fcfa) }\end{array}$ & Farine & 67 \\
$\begin{array}{l}\text { Bénéfice brut } \\
\text { réalisé par rapport } \\
\text { au gain de poids (Fcfa) }\end{array}$ & Granulé & 96816 \\
$\begin{array}{l}\text { Bénéfice brut } \\
\text { réalisé par rapport }\end{array}$ & Farine & 61584 \\
à l'aliment farineux (Fcfa) & Farine & 59013 \\
$\begin{array}{l}\text { Bénéfice réalisé par } \\
\text { rapport à un lapin (Fcfa) }\end{array}$ & Granulé & 76652 \\
& & - \\
\hline
\end{tabular}

NB : le prix de vente du kilogramme de poids vif de lapin était à 1000 Fcfa

\section{Indices de consommation}

Très peu d'études ont porté sur les indices de consommation des lapins, toutes races confondues, en Afrique subsaharienne. Toutefois, de manière classique, l'indice de consommation s'accroît avec l'âge de l'animal. Dans une étude réalisée sur des animaux de la même race, l'aliment farineux de même composition que celle utilisée dans la présente étude a donné un indice de consommation hebdomadaire qui a augmenté de 1,9 à 5,8, de la première à la huitième semaine d'engraissement, avec une moyenne hebdomadaire de 3,3 pour les huit semaines d'engraissement (10). La moyenne de l'indice de consommation pour toute la période expérimentale a été de 3,74 pour les animaux nourris à l'aliment granulé contenant 5 p. 100 de mélasse et de 3,61 pour les animaux nourris à l'aliment granulé de même composition que celui de la présente étude (9). Ces différentes valeurs de l'indice de consommation sont proches de celles obtenues dans la présente étude.

\section{Impacts sur la santé des lapereaux}

Les cas de pathologies observées au cours de l'expérimentation n'étaient pas liés aux coccidies mais à l'aliment en raison du traitement anticoccidien réalisé au début de l'expérimentation dans chaque unité d'élevage. Le fort taux de mortalité constaté parmi les animaux nourris à l'aliment farineux pourrait être expliqué par le fait qu'avec cet aliment les lapereaux ont trié et rejeté les parties fibreuses nécessaires au bon fonctionnement du tube digestif. Cette alimentation, déficiente en fibres, aurait favorisé les entérites. Le taux de mortalité a été de 31,7 p. 100 lorsque l'aliment était pauvre en fibres (19,1 p. 100 de lignocellulose), contre
11,5 p. 100 lorsqu'il était riche en fibres (23,9 p. 100 de lignocellulose). De même, le taux de morbidité a été de 38,5 p. 100 chez les lapereaux nourris avec l'aliment pauvre en fibres, contre 18,5 p. 100 pour l'aliment riche en fibres (12). Bennegadi et coll. (2) ont étudié l'incidence d'une forte baisse du niveau des fibres alimentaires (19 à 9 p. 100 de lignocellulose, respectivement pour l'aliment témoin $\mathrm{S}$ et déficient en fibres D) sur le statut sanitaire et la mortalité des lapereaux en engraissement. Ils ont rapporté que les diarrhées étaient le symptôme le plus fréquemment observé dans les entérites provoquées par la réduction du taux de fibres ( 90 p. 100 des cas), et que les lésions digestives étaient essentiellement une dilatation du segment caeco-colique (30 p. 100 de cas) et une liquéfaction du contenu digestif intestinal et caecal (60 p. 100 de cas). Ils ont aussi relevé un taux de mortalité de 25 p. 100 chez les lapins nourris avec le régime déficient en fibres D (période 28-70 j d'âge), contre 9 p. 100 chez ceux nourris avec le régime témoin $\mathrm{S}$. Enfin, selon Auvergne et coll. (1), 16 lapins sur 60 sont morts de troubles digestifs avant la fin des 49 jours d'essai avec la mouture fine, contre 8 lapins sur 60 seulement avec la mouture grossière ; les 16 lapins morts de troubles digestifs avec le broyage fin étaient tous atteints de diarrhées, mais quatre des huit lapins morts avec le broyage grossier avaient un blocage du caecum (parésie caecale), et les quatre autres des symptômes de diarrhées. Au niveau de la santé de l'animal, un apport en lignocellulose paraît efficace pour réduire la fréquence des troubles digestifs et la mortalité pendant la période d'engraissement des lapins (5).

Par ailleurs, la poussière de la farine inhalée par les animaux pourrait être à l'origine des complications pulmonaires, puisque le taux élevé des mortalités lié aux maladies respiratoires a été uniquement constaté dans le lot des animaux nourris à l'aliment farineux dans les trois fermes.

\section{CONCLUSION}

L'aliment granulé a permis d'obtenir des performances nettement meilleures chez les lapins que la forme farineuse correspondante et de réduire de 13 à 15 jours la durée d'engraissement. L'aliment granulé a été plus consommé, moins gaspillé et a présenté un meilleur indice de consommation que l'aliment farineux ; il a aussi conduit à une mortalité inférieure. Par rapport à l'aliment farineux, une marge bénéficiaire de 245 Fcfa par lapin a été obtenue à l'issue de cette étude, confirmant la rentabilité économique de l'aliment granulé.

\section{BIBLIOGRAPHIE}

1. AUVERGNE A. BOUYSSOU T., PAIRET M., BOUILLIER OUDOT M., RUCKEBUSCH Y., CANDAU M., 1987. Diet type, grinding size and anatomical and functional characteristics of the proximal digestive tract in rabbits. Reprod. Nutr. Dev., 27: 755-768.

2. BENNEGADI N., GIDENNE T., LICOIS L., 2001. Impact of fibre deficiency and sanitary status on non-specific enteropathy of the growing rabbit. Anim. Res., 50: 401-413.

3. DEPRES E., VARO H., MATHERON G., XANDE A., 1991. Essai comparatif de deux aliments granulés utilisés en Guadeloupe sur les performances de croissance et le rendement à l'abattage de lapins croisés néozélandais blancs x californiens. Cuni-Sci., 7 : 23-27.

4. JAGO A.Y., KPODEKON T.M., 2007. Le guide pratique de l'éleveur de lapins en Afrique de l'Ouest, 2e édn. Corronsac, France, association Cuniculture. http://www.cuniculture.info/ Docs/Elevage/Tropic-01.htm

5. GIDENNE T., 1996. Conséquences digestives de l'ingestion de fibres et d'amidon chez le lapin en croissance : vers une meilleure définition des besoins. Prod. Anim., $9: 243-254$. 
6. GOBY P., ROCHON J., 1990. Utilisation d'un aliment fermier chez le lapin à l'engraissement : digestibilité et impact du tri alimentaire. In : $5^{\text {es }}$ Journées Recherche cunicole, Paris, France, 12-13 déc. 1990.

7. KPODEKON M., LEBAS F., DJAGO Y., COUDERT P., 1998. Relative efficiency of local meal concentrate pelleted feed for fattening rabbits in tropical conditions. Interaction with rabbit origin. World Rabbit Sci., 6: 291 287.

8. KPODEKON T.M., YOUSSAO A.K.I., DJOGBENOU I., DJAGO Y. 2005. Performances de croissance et viabilité des lapereaux nourris avec un aliment granulé à l'engraissement. Revue Afr. Santé Prod. Anim., 3 : 222-226.

9. KPODEKON T.M., YOUSSAO A.K.I., TOSSOU C.M., DJAGO A.Y., COUDERT P., 2008. Effects of molasses incorporation in rabbit fattening diet on growth performances. In: Proc. 9th World Rabbit Congress,

Verona, Italy, 10-13 June 2008, p. 711-715.

\section{Summary}

Kpodékon M., Youssao A.K.I., Koutinhouin G.B., Baba I.L., Dessou J.M., Djago Y. Effect of Diet Pelleting on Growth, Feed Efficiency and Viability of the Rabbit in a Tropical Breeding Environment

One hundred and forty-four young rabbits were used from weaning (34-36 days old) to study growth, feed efficiency and viability in relation to the diet presentation: pelleted or as a flour. The experiment was carried out in three farms (CECURI, GAVA and AHINADJE), with 48 young rabbits in each farm, divided into two groups of 24 animals: an experimental group fed a pellet diet and a control group fed the same diet but in a flour form. Weights and feed intake were measured weekly during the eight-week fattening period, and mortality was checked daily. During fattening (56 days), the young rabbits fed the pelleted diet had a higher $(\mathrm{P}<0.001)$ growth rate $(24$ $\mathrm{g} / \mathrm{d})$ than those fed the flour diet $(22 \mathrm{~g} / \mathrm{d})$. During the first four weeks, the pelleted diet was more consumed $(+18 \%)$, less rejected $(-77 \%)$, with a lower consumption index $(-20 \%)$ than the flour diet $(\mathrm{P}<0.001)$. The consumption index $(5.2)$ and feed intake (92 g) were similar during the last four weeks $(\mathrm{P}>0.05)$. On the other hand, the pelleted diet was less rejected $(\mathrm{P}<0.001)$ than the flour diet $(1 \mathrm{~g}$ vs $4 \mathrm{~g})$. Finally, the mortality rate was lower $(15.28 \%)$ in the groups of young rabbits that received the pelleted diet than in those that received the flour diet $(46 \%)$

Keywords: Rabbit - Compound feed - Growth - Viability Benin.
10. KPODEKON T.M., YOUSSAO A.K.I., KOUTINHOUIN G.B., FAYOMI J., FAGBOHOU A., DJAGO Y., 2009. Substitution du tourteau de palmiste par le tourteau de tournesol dans l'alimentation des lapins à I'engraissement. Livest. Res. Rural Dev., 21. http://www.Irrd.org//rrd21/6/ kpod21092.htm

11. SAS, SAS/STAT, 1996. User's guide, Vers. 6. Cary, NC, USA, SAS Inst. 12. XICCATO G., TROCINO A., CARRARO L., FRAGKIADAKIS M., MAJOLINI D., 2008. Digestible fibre to starch ratio and antibiotic treatment time in growing rabbits affected by epizootic rabbit enteropathy. In: Proc. 9th World Rabbit Congress, Verona, Italy, 10-13 June 2008, p. 847-851.

Accepté le 10.02.2010

\section{Resumen}

Kpodékon M., Youssao A.K.I., Koutinhouin G.B., Baba I.L., Dessou J.M., Djago Y. Efecto de granulados sobre los rendimientos de crecimiento, eficacidad alimenticia y viabilidad de conejos bajo condiciones de crianza tropical

Se utilizaron 140 conejos de raza local a partir del destete (entre 34 y 36 días de edad) para analizar el crecimiento, la eficiencia alimenticia y la viabilidad, en función de la presentación del alimento: harina o granulados. El experimento se desarrolló en tres fincas (Cecuri, Gava y Ahinadjè), con 48 conejos por finca, repartidos en dos lotes de 24 conejos: un lote experimental alimentado con un alimento granulado y un lote control alimentado con el mismo alimento, pero en forma de harina. El peso y el consumo se midieron en forma hebdomadaria, durante las ocho semanas de engorde y la mortalidad fue controlada cotidianamente. Durante la duración del engorde (56 días), los conejos alimentados con el alimento granulado presentaron una velocidad de crecimiento $(24 \mathrm{~g} / \mathrm{d})$ más elevada $(\mathrm{P}<0,001)$ que la de los conejos alimentados con el alimento harinoso (22 g/d). Durante las cuatro primeras semanas, el alimento granulado fue más consumido (+ 18\%), menos rechazado (- 77\%), con un índice de consumo más bajo $(-20 \%)$ que el alimento harinoso $(\mathrm{P}<0,001)$. El índice de consumo $(5,2)$ y las cantidades de alimento consumidas (92 g) fueron idénticas durante las cuatro últimas semanas $(\mathrm{P}>0,05)$. En cambio, el alimento granulado fue menos rechazado $(\mathrm{P}<0,001)$ que el alimento harinoso (1g vs $4 \mathrm{~g})$. Finalmente, en los lotes de conejos alimentados con el granulado, la tasa de mortalidad fue de $15 \%$, contra $46 \%$ en los conejos alimentados con el alimento harinoso.

Palabras clave: Conejo - Pienso compuesto - Crecimiento Viabilidad - Benin. 